\title{
Laparoscopic treatment of type III and IV hiatal hernia - authors' experience
}

\author{
Marcin Migaczewski, Agata Grzesiak-Kuik, Michał Pędziwiatr, Andrzej Budzyński \\ $2^{\text {nd }}$ Department of General Surgery, Medical College Jagiellonian University, Krakow, Poland
}

Videosurgery Miniinv 2014; 9 (2): 157-163

DOI: $10.5114 /$ wiitm.2014.41625

\begin{abstract}
Introduction: There are four types of hiatal hernias, and diagnosis is established on the basis of gastroscopy in the majority of cases. Type III represents a mixed type in which the abdominal esophagus as well as the gastric cardia and fundus protrude into the thorax through the pathologically widened esophageal hiatus. Type IV, the so-called upside down stomach, can be considered an evolutionary form of type III, and refers to herniation of nearly the whole stomach (except for the cardia and pylorus) into the thorax. Types III and IV of hiatal hernias represent a group of rare diaphragmatic defects; thus, most centers do not possess considerable experience in their treatment. Frequently, laparoscopic treatment is implemented, although, according to some authors, conversion to laparotomy, thoracotomy, or thoracolaparotomy is necessary in selected cases.

Aim: To analyze the outcomes of laparoscopic treatment of the largest hiatal hernias, i.e. type III and IV hernias.

Material and methods: A total of 25 patients diagnosed with type III and IV hiatal hernia were included in further analysis.

Results: As many as 19 out of 25 patients (76\%) assessed the outcome of the surgery as evidently positive and reported marked improvement in the quality of life.

Conclusions: The laparoscopic technique constitutes an excellent and safe method of repair of even the most complex defects in the esophageal hiatus. Therefore, the minimally invasive technique combined with an anti-reflux procedure should be the method of choice in patients with type III and IV hernia.
\end{abstract}

Key words: laparoscopy, type III and IV hiatal hernia, Nissen fundoplication.

\section{Introduction}

There are four types of hiatal hernias, and diagnosis is established on the basis of gastroscopy in the majority of cases (Photo 1). Sliding hernia (type I), in which the abdominal esophagus and a variable portion of the cardia protrude into the thorax, represents the vast majority of cases (90\%). There are no indications for pharmacotherapy, and especially surgical treatment, in asymptomatic cases. However, some patients report signs of gastroesophageal reflux disease (GERD), which can substantiate their qualification for surgery. In the case of paraesophageal hernia (type II), the gastric fundus is herniated, while the abdominal esophagus and cardia remain in their normal (subdiaphragmatic) localization. Types III and IV constitute approximately $5 \%$ of all hiatal hernias [1]. Type III represents a mixed type in which the abdominal esophagus as well as the gastric cardia and fundus protrude into the thorax through pathologically widened esophageal hiatus. Type IV, the so-called upside down stomach, can be considered an evolutionary form of type III, and refers to herniation of nearly the whole stomach

\section{Address for correspondence:}

Marcin Migaczewski MD, PhD, $2^{\text {nd }}$ Department of General Surgery, Medical College Jagiellonian University, 21 Kopernika St, $31-501$ Krakow, Poland, phone: +48 1242482 00, e-mail: marcinmigaczewski@onet.pl 


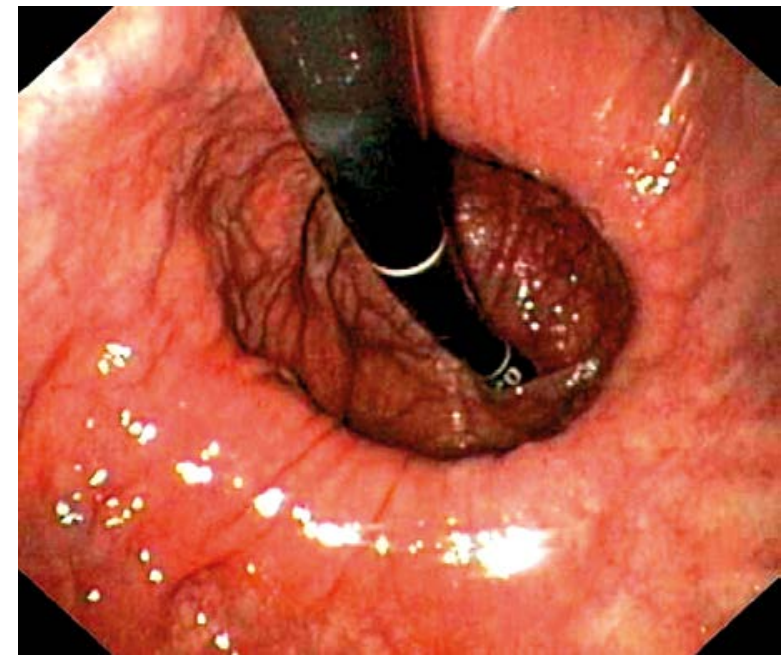

Photo 1. Gastroscopy - hiatal hernia type III

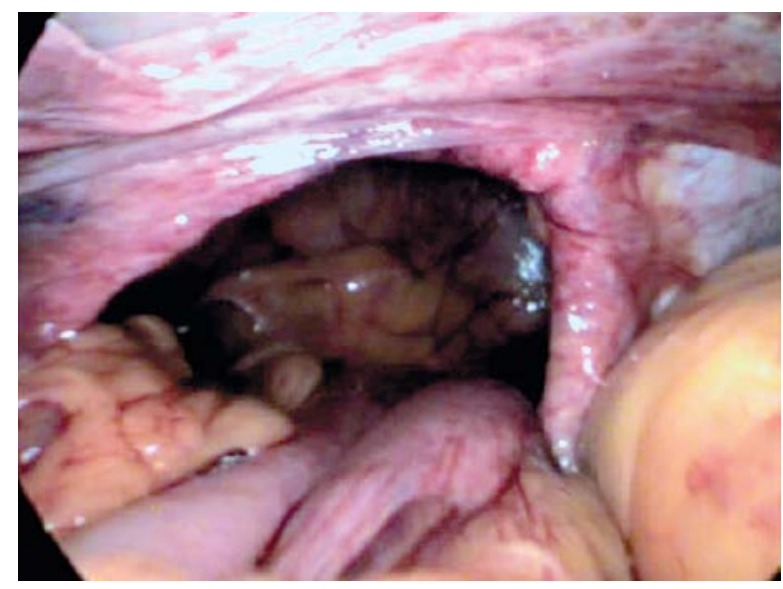

Photo 3. Hiatal hernia type IV - intraoperative view

(except for the cardia and pylorus) into the thorax (Photos 2 and 3). In the case of the largest defects of esophageal hiatus, other organs, such as intestinal convolutions or spleen, can also protrude into the thorax.

Signs extending beyond the spectrum of reflux disease symptoms, and the risk of entrapment, constitute the main indications for surgical treatment in patients with type II, III, and IV hiatal hernias. Patients present with pain, frequently of stenocardial character, swallowing disorders, and complications resulting from the compression of thorax organs. Cases of entrapment have quite dramatic clinical manifestation and require emergency surgery, which is not infrequently associated with significant morbidity.

Types III and IV of hiatal hernias represent a group of rare diaphragmatic defects; thus, most centers do

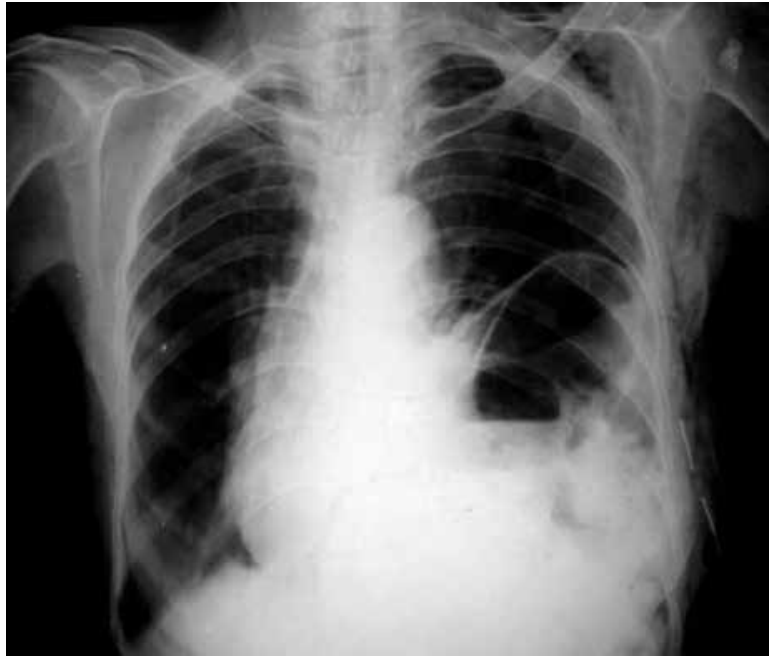

Photo 2. X-ray of the chest - hiatal hernia type IV

not possess considerable experience in their treatment. As a result, an individualized attitude predominates in surgical treatment of such cases. Moreover, some authors postulate that such cases should be qualified for open surgery by default. Nevertheless, irrespective of approach, surgical treatment of large hernias can be challenging. The main technical difficulties pertain to relocation of hernial contents into the abdominal cavity, dissection of adhesions frequently involving both the abdomen and the mediastinum, repair of large diaphragmatic defects, and sometimes also considerable shortening of the esophagus. Consequently, this spectrum of problems extends well beyond the scope of typical anti-reflux surgery during which repair of usually small sliding (type I) hernia remains the key problem.

\section{Aim}

The aim of this study was to analyze the outcomes of laparoscopic treatment of the largest hiatal hernias, i.e. type III and IV hernias.

\section{Material and methods}

The study included patients of the $2^{\text {nd }}$ Department of Surgery Medical College Jagiellonian University, who were treated surgically due to large hiatal hernias (defects wider than $5 \mathrm{~cm}$ ) during the last 10 years. A total of 25 patients diagnosed with type III and IV hiatal hernia were included in further analysis. The diagnosis was based on gastroscopy and barium contrast esophageal radiography. Moreover, computed tomography of the chest was conducted 
in some patients in order to determine detailed anatomical conditions. Type III and IV hiatal hernia was diagnosed in 15 and 10 patients, respectively.

Dyspnea ( $n=10,40 \%$ ) was the predominant symptom in the qualified patients. Signs of gastroesophageal reflux disease, mostly in the form of heartburn, were reported by 8 patients (32\%). Substernal pain was the main symptom in 5 patients (20\%); 7 out of 25 patients complained of dyspepsia prior to the surgery.

All patients were qualified for laparoscopic plasty of the esophageal hiatus. The procedure was carried out by three operators with the patient placed in the so-called Fowler's (reverse Trendelenburg) position (the patient is placed in the supine position and the lower limbs are abducted). Initially, a manual fan retractor inserted through a $10 \mathrm{~mm}$ trocar was used to retract the left hepatic lobe. Recently, it was replaced by an automatic Nathanson retractor, mounted onto the operating table and inserted into the abdominal cavity via the opening following a $5 \mathrm{~mm}$ trocar.

The procedure commenced with the dissection of the diaphragmatic crura. Upon adhesiolysis, the esophagus was mobilized at a level enabling its free downward retraction and reconstruction of at least a $2 \mathrm{~cm}$ abdominal segment. Simultaneously, the stomach protruding into the thorax was dissected. Upon opening the omental sac, short gastric vessels were cut with a harmonic knife in order to fully mobilize the gastric fundus.

The second stage comprised plasty of the hiatal ring. In most patients, diaphragmatic crura were connected with non-absorbable 2.0 braid-type sutures. In the first few years following the implementation of the laparoscopic technique, diaphragmatic crura of patients with large hiatal hernias were connected with a combination of single and Z-type sutures. In the last few years, however, only single sutures were used in most patients. In 5 patients (20\%), continuous suture was applied onto the diaphragmatic crura. While diaphragmatic crura were connected posteriorly to the esophagus (so-called posterior cruroplasty) in 22 patients (88\%), we decided on anterior cruroplasty in 3 subjects (12\%) due to considerable weakening of the diaphragmatic crura posterior to the esophagus.

An intraoperative decision on the use of surgical mesh was made during primary surgery of 1 patient due to the defibration of weakened diaphragmatic crura during an attempt to connect them; polypro- pylene mesh was used. In another female patient, the decision on mesh implantation was made during reoperation due to recurrent hernia with migration of the fundoplication band. Conversion from laparoscopy to thoracotomy was required in 1 case due to planar adhesions of the stomach, which was protruding almost entirely into the thorax.

Although the typical signs of reflux disease were not frequently documented in the analyzed group, we followed the general rule of completing each hernioplasty with Nissen fundoplication. The fundoplication band formed during this procedure additionally stabilized the reconstructed abdominal segment of the esophagus.

\section{Results}

The study included patients who were operated on between January $1^{\text {st }}, 2003$ and December $31^{\text {st }}$, 2012. Mean duration of follow-up was 57 months (range: 4-118 months). Each patient was subjected to hiatal hernioplasty and an anti-reflux procedure in the form of laparoscopic Nissen fundoplication.

The average duration of the surgery was $147 \mathrm{~min}$ (range: 60-270 $\mathrm{min}$ ), and the average duration of hospital stay amounted to 4 days (range: 2-7 days).

No perioperative mortality was documented and emergency reoperation was not required in any patient. The right or left pleura was injured in $3(12 \%)$ and 2 (8\%) patients, respectively. All pleural injuries were closed with single sutures; conversions to other type of surgery or drainage were not needed.

Six months after the primary procedure, recurrent hernia along with migration of the fundoplication band into the thorax was documented in 1 female patient (4\%). The patient was re-operated laparoscopically on an elective basis. A decision on using polypropylene mesh was made intraoperatively due to considerable defibration of diaphragmatic crura.

As many as 19 out of 25 patients (76\%) assessed the outcome of the surgery as evidently positive and reported marked improvement in the quality of life. Dyspnea and substernal pain resolved in all patients. Amongst the patients in whom the symptoms of GERD predominated prior to the surgery, there was only $1(4 \%)$ in whom these problems persisted postoperatively.

The feeling of early satiety ( $n=15,60 \%$ ) predominated amongst postoperative adverse events, 
followed by transient postprandial abdominal pain resolving up to 2-6 months after surgery, and dysphagia persisting longer than 8 weeks after the procedure $(n=5,20 \%)$. Neither reoperation nor endoscopic treatment due to the signs of postoperative esophageal stenosis was required in any patient.

\section{Discussion}

Laparoscopic surgery has assumed the dominant position in the surgical treatment of benign conditions of the gastroesophageal junction. Precise exposure of the esophageal hiatus area is possible due to a different position of the surgical team as compared to classical surgery, as well as due to the properties of the laparoscopic video channel itself. A different angle of vision, obtained by the use of the so-called oblique laparoscopes among others, dramatically changes possibilities of visualizing the area of diaphragmatic crura connection, even under unfavorable anatomical conditions (e.g. in obese patients with low costal arches). Comparison between laparotomy and laparoscopy in terms of possible visualization of the esophageal hiatus area, and especially the mediastinum, evidently favors less invasive techniques (Figure 1). Also, the possibility of stable retraction of the left hepatic lobe with devices mounted to an operating table, such as the Nathanson retractor, is important for the comfort of surgical access. The laparoscopic approach is especially valu-

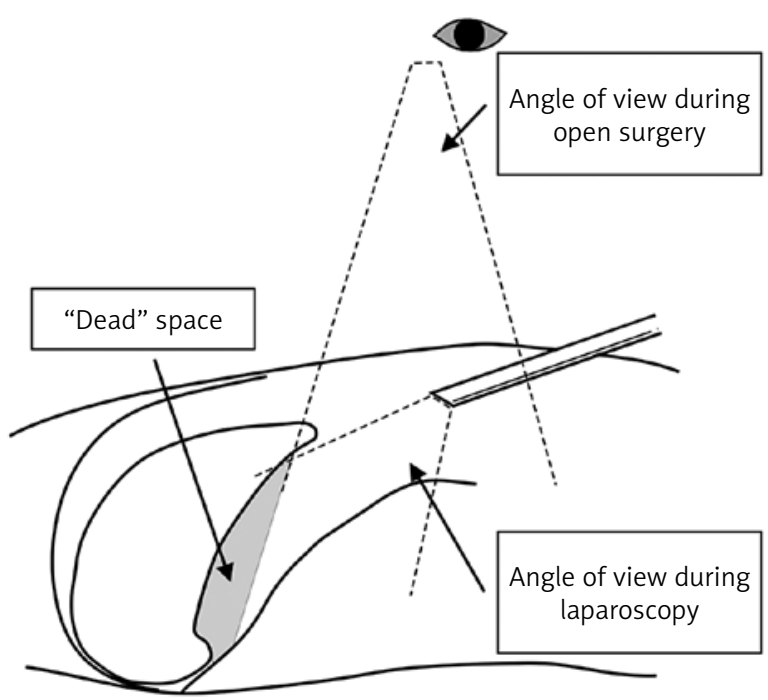

Figure 1. Comparison between laparotomy and laparoscopy in terms of visualization of the esophageal hiatus area able during the stage of mediastinal dissection. Excellent exposure and precise laparoscopic tools enable safe dissection of periesophageal tissues within a bloodless surgical field. Reaching the plateau of the learning curve is associated with improved accessibility to the level of inferior pulmonary veins, or even tracheal bifurcation.

At present, less invasive surgery constitutes a "gold standard" in the treatment of sliding (type I) hiatal hernias [2, 3]. Signs of GERD constitute the principal indication for surgery in patients with this condition. Laparoscopic Nissen fundoplication, including plasty of hiatal hernia, remains the technique of choice and usually is not associated with any greater difficulties [4]. However, qualification of patients from this group for surgical treatment is frequently questionable. Apart from the prevention of esophageal cancer in patients with Barrett's esophagus (BE), the qualification is based on less harmful signs which diminish the patient's quality of life to a various extent. In view of highly satisfactory outcomes of the conservative treatment of GERD, the decision on surgery is not infrequently based solely on preferences of a given patient.

The situation is dramatically different in the case of indications for surgical treatment in patients with type II, III, and IV hernias. Due to the risk of entrapment and manifestations associated with the compression of thoracic structures, leading to dyspnea and stenocardial pain, the qualification is based on considerably stricter criteria. According to available literature, the prevalence of mixed hiatal hernias (type III and IV) is estimated at only about $5 \%$. In some authors' opinion, the mixed hernias represent more advanced stages of diaphragmatic defects associated with sliding hernia. Type III and IV hernias corresponded to approximately $8 \%$ of all hernias observed in our patients qualified for laparoscopic Nissen fundoplication. The lack of GERD manifestation in most of the cases from this group determines a different protocol of preoperative examination and qualification for treatment. According to various authors, the prevalence of heartburn in patients with mixed hernias ranges between 19\% (Myers) and $67 \%$ (Gantert) [5, 6]. We documented signs of GERD with predominant heartburn in $8(32 \%)$ of our patients.

Taking into account relatively rare manifestation of GERD in most patients with large hiatal hernias, either $\mathrm{pH}$-metry or $\mathrm{pH}$-impedance alone is postulat- 
ed to be redundant during qualification for surgical treatment. Also, esophageal manometry does not give reliable results, especially in the case of large hernias. According to some authors who consider the possibility of partial fundoplication, impaired motility of the esophageal body plays some role during the qualification process. However, in our opinion, a decreased peristaltic esophageal wave constitutes a constant component of this condition resulting from herniation of the esophagus into the thorax and resultant decrease in its tonus, being a determinant of normal contractility. Contrast radiographic examination of the esophagus in patients with type III and IV hernia is ascribed greater importance than in the case of individuals with sliding hernias. Usually, this examination quite precisely identifies the part of the stomach which has protruded into the thorax and enables the estimation of esophageal length. Computed tomography with an oral contrast agent is recommended whenever transposition of other organs, e.g. spleen or colon, is suspected. Furthermore, this examination is also vital in the case of recurrent hernia or suspicion of post-traumatic injury of diaphragmatic crura. According to a controversial opinion, revealing large defect in this region can constitute a relative contraindication to laparoscopic surgery.

Due to the low prevalence of the condition, most centers lack extensive experience in its treatment; as a result, technical details of the surgery still raise some controversies. Some authors point to the necessity of dissecting the hernial sac [7]. In our opinion, this procedure is not difficult and allows for considerably better assessment of the mediastinal anatomy. On the other hand, some reports suggest that the dissection of the hernial sac is associated with increased risk of pleural injury or even injury to the lung parenchyma or pericardium. This can occur especially in older patients with a long-term history of hernia and large diaphragmatic defects. Nevertheless, despite using this technique, we did not document increased morbidity in our patients.

Our experience suggests superiority of non-absorbable sutures in repair of the hernial ring. In patients operated on at our center, diaphragmatic crura were connected with single sutures posteriorly to the esophagus (posterior cruroplasty). An attempt to use continuous sutures was made in 5 patients, but we did not prove the superiority of this technique. Connecting diaphragmatic crura anteriorly to the esophagus (anterior cruroplasty) is referred to as the "last resort" in the literature. We used this method in only three patients, always with a good result. We suggest considering such a technique of hernial ring repair in the case of considerable defibration of the crura in their posterior parts.

Some authors emphasize the advantages of fixing the proximal portion of the stomach at the region of diaphragmatic crura in order to stabilize the fundoplication band and the reconstructed angle of His. In our opinion, any simultaneous form of gastropexy (anterior, posterior) is unjustified and inconsistent with the rules of the so-called floppy Nissen technique.

The use of surgical mesh for the repair of large hiatal hernias still remains controversial. The possibility of supporting defective and frequently defibrating diaphragmatic crura, which not infrequently can barely be connected with sutures, represents one argument for such an approach. However, in our opinion, the use of synthetic implants is rarely necessary and refers mostly to cases of recurrent hernia. In the case of primary surgery, meshes should be used on a highly individualized basis. Amongst our patients, we decided to support defibrated diaphragmatic crura with mesh in the course of primary surgery in only one subject. Alternatively, in such cases diaphragmatic crura can be connected anteriorly to the esophagus (anterior cruroplasty) as they are usually less exposed to stretching in this location. There is no agreement between the supporters of surgical mesh implantation with regards to the safest type of this material [8]. While some authors emphasize the advantages of modern biological semi- or fully absorbable meshes, others have documented safe use of PTFE or polyester implants [9]. Nevertheless, complications reported in the literature, associated with migration of the mesh into the esophageal lumen, and excellent outcomes of treatment without the implantable meshes, are reflected by a diminishing group of enthusiasts of this method [10].

Shortening of the esophagus is another important issue. It requires respective gastroplasty in rare cases, most commonly with the Collis method [11]. Although this method is considered an effective technique of esophageal elongation, there are some reports of dysfunction of the anti-reflux barrier resulting from this procedure, mostly due to impaired self-clearance of the distal newly formed esophageal segment [12]. In our opinion, high mobilization of 
the esophagus inside the thorax is sufficient for its elongation in the vast majority of cases. We did not implement any form of gastroplasty in our patients, even in cases of a significantly shortened esophagus.

The less invasive form of Nissen fundoplication constitutes the presently established standard of treatment in GERD patients [13-15]. According to many authors, the possibility of co-existing signs of gastroesophageal reflux disease $[5,16]$ and the risk of recurrent hernia constitute the main indication for simultaneous fundoplication in patients with type III and IV hernia [17]. In our opinion, anti-reflux treatment plays an unquestioned role in the management of patients with large hiatal hernias. The fundoplication band formed during the procedure allows for additional stabilization of the stomach and abdominal esophagus within the peritoneal cavity. According to many reports, the risk of manifestation of GERD in patients operated on due to hiatal hernia without simultaneous fundoplication can reach up to $65 \%$. On the other hand, there are some reports on the increased risk of postoperative dysphagia, especially after so-called complete $\left(360^{\circ}\right)$ fundoplication $[18,19]$. Some authors do not accept simultaneous anti-reflux treatment in patients without typical signs of GERD and recommend selective qualification for simultaneous anti-reflux surgery. This attitude seems completely unjustified in view of our findings as they suggest that simultaneous fundoplication should be obligatorily recommended.

Many authors emphasize that surgical anatomy of patients with type III and IV hernia is different than in the case of smaller diaphragmatic defects. The necessity of more extensive dissection of tissues, especially in the mediastinum, is associated with higher incidence and severity of intraoperative complications. The literature contains evidence of intraoperative injuries to virtually all anatomical structures located close to the esophageal hiatus: pleura, esophagus, aorta, thoracic duct, spleen, and stomach. We did not document any complications requiring reoperation in our material. Pleural injuries, leading to pneumothorax and requiring drainage, predominated amongst observed intraoperative complications. Undoubtedly, the recurrence of hernia after surgical treatment should be listed amongst potential complications. We documented such a case in 1 female patient (4\%). The recurrence rates of hernia reported by other authors are highly variable, ranging from $5 \%$ to more than $20 \%$ [20].
In most cases, the recurrence of hernia remains asymptomatic and is diagnosed endoscopically or on radiography; this delays the implementation of causative treatment [21].

Despite different rules of qualification for surgery, evaluation of potential improvement in the quality of life due to the alleviation of initial symptoms remains an important therapeutic outcome. Similarly to previous research, our study revealed apparent improvement in the quality of life of most treated patients. Virtually all symptoms resolved immediately after the surgery in all of our subjects. Not surprisingly, we documented an excellent outcome with regards to the attenuation of reflux disease symptoms. These results and benefits associated with the use of a less invasive technique were reflected by highly satisfactory scores of subjectively assessed quality of life of surgically treated patients.

\section{Conclusions}

The laparoscopic technique constitutes an excellent and safe method of repair of even the most complex defects in the esophageal hiatus. Therefore, the minimally invasive technique combined with an anti-reflux procedure should be the method of choice in patients with type III and IV hernia.

\section{References}

1. Skinner DB, Belsey RH. Surgical management of esophageal reflux and hiatus hernia. Long-term results with 1030 patients. J Thorac Cardiovasc Surg 1967; 53: 33-54.

2. Wróblewski T, Skalski M, Ziarkiewicz-Wróblewska B, et al. New antireflux surgical technique in GERD treatment. Videosurgery Miniinv 2007; 2: 139-44.

3. Wróblewski T, Skalski M, Ziarkiewicz-Wróblewska B, et al. Progress in surgical treatment of reflux disease. Videosurgery Miniinv 2006; 1: 121-4.

4. Rembiasz K, Migaczewski M, Budzyński A, et al. Expression of cyclooxygenase- 2 in the mucosa of the gastroesophageal junction in patients with Barrett's oesophagus - the results of ablation therapy with argon plasma coagulation and laparoscopic Nissen fundoplication. Videosurgery Miniinv 2010; 5: 45-52.

5. Myers G, Harms B, Starling J. Management of paraesophageal hernias with a selective approach to antireflux surgery. Am J Surg 1995; 170: 375-80.

6. Gantert WA, Patti MG, Arcerito M, et al. Laparoscopic repair of paraesophageal hiatal hernias. J Am Coll Surg 1998; 186: 428-32.

7. Rosenthal RJ, Jones DB. Laparoscopic hiatal hernia repair. Bariatric Times 2012; 9: 8-9. 
8. Furnée E, Hazebroek E. Mesh in laparoscopic large hiatal hernia repair: a systematic review of the literature, Surg Endosc 2013; 27: 3998-4008

9. Schmidt E, Shaligram A, Reynoso JF, et al. Hiatal hernia repair with biologic mesh reinforcement reduces recurrence rate in small hiatal hernias. Dis Esophagus 2014; 27: 13-7.

10. Carpelan-Holmström M, Kruuna O, Salo J, et al. Late mesh migration through the stomach wall after laparoscopic refundoplication using a dual-sided PTFE/ePTFE mesh. Hernia 2011; 15: 217-20.

11. Mori T, Nagao G, Sugiyama M. Paraesophageal hernia repair. Ann Thorac Cardiovasc Surg 2012; 18: 297-305.

12. Mor A, Lutfi R, Torquati A. Esophageal acid-clearance physiology is altered after Nissen-Collis gastroplasty. Surg Endosc 2013; 27: 1334-8.

13. Dąbrowski WP, Szczepanik AB, Misiak A, Pielaciński K. Radiofrequency ablation in the management of Barrett's esophagus - preliminary own experience. Videosurgery Miniinv 2013; 8: 107-11.

14. Migaczewski M, Pędziwiatr M, Matłok M, Budzyński A. Laparoscopic Nissen fundoplication in the treatment of Barrett's esophagus - 10 years of experience. Videosurgery Miniinv 2013; 8: 139-45.

15. Hermann J, Kościński T, Malinger S, et al. Strangulation of the stomach and the transverse colon following laparoscopic esophageal hiatal hernia repair. Videosurgery Miniinv 2012; 7: 311-4.

16. Fuller CB, Hagen JA, DeMeester TR, et al. The role of fundoplication in the treatment of type II paraesophageal hernia. J Thorac Cardiovasc Surg 1996; 111: 655-61.

17. Casabella F, Sinanan M, Horgan S, et al. Systematic use of gastric fundoplication in laparoscopic repair of paraesophageal hernias. Am J Surg 1996; 171: 485-9.

18. Draaisma WA, Gooszen HG, Tournoij E, et al. Controversies in paraesophageal hernia repair: a review of literature. Surg Endosc 2005; 19: 1300-8.

19. Furnée EJB, Draaisma WA, Gooszen HG, et al. Tailored or routine addition of an antireflux fundoplication in laparoscopic large hiatal hernia repair: a comparative cohort study. World J Surg 2011; 35: 78-84.

20. Antoniou SA, Antoniou GA, Koch OO, et al. Lower recurrence rates after mesh-reinforced versus simple hiatal hernia repair: a meta-analysis of randomized trials, Surg Laparosc Endosc Percutan Tech 2012; 22: 498-502.

21. Parameswaran R, Ali A, Velmurugan $\mathrm{S}$, et al. Laparoscopic repair of large paraesophageal hiatus hernia: quality of lile and durability. Surg Endosc 2006; 20: 1221-4.

Received: 8.08.2013, accepted: 11.10.2013. 